Original Article

\title{
Modelling spatial patterns of misaligned disease data: An application on measles incidence in Namibia
}

\author{
D. Ntirampeba ${ }^{a, *}$, I. Neema ${ }^{\text {, }}$ L.N. Kazembe ${ }^{c}$ \\ a Department of Mathematics and Statistics, Namibia University of Science and Technology, Windhoek 264, Namibia \\ ${ }^{\mathrm{b}}$ Namibia Statistics Agency (NSA), Windhoek 264, Namibia \\ ${ }^{\mathrm{c}}$ Department of Statistics and Population Studies, University of Namibia, Windhoek 264, Namibia
}

\section{A R T I C L E I N F O}

\section{Article history:}

Received 18 November 2016

Accepted 24 January 2017

Available online 6 April 2017

\section{Keywords:}

Hierarchical Bayesian model

Misalignment

Measles

Namibia

Spatial modelling

\begin{abstract}
A B S T R A C T
Introduction: Quite often disease data are available in aggregated formats mostly to maintain confidentiality. This leads to a misalignment problem when the goal is to analyze risk at a different level of spatial resolution different from the original administrative level where data were available. Objective: To estimate and map the risk of measles at a sub-region level in Namibia using data obtained at a regional level.

Methods: Using measles data from Namibia for the period 2005-2014, both multi-step and direct approaches were applied to correct for misalignment. Subsequently, ecological Bayesian regression models were fitted and compared.

Results: Results show that the variables standardized birth rate, counts of measles cases for previous year, unemployment rate and proportion of vaccinated children against measles by age 12 months were significant determinants of measles risk. Constituencies having elevated measles risk were identified mostly in the northern corridor with Angola.

Conclusion: We recommend that relevant authorities should make geographical target intervention and redesign prevention and control strategies based on these findings.
\end{abstract}

(C) 2017 INDIACLEN. Published by Elsevier, a division of RELX India, Pvt. Ltd. All rights reserved.

\section{Introduction}

Measles is a disease caused by a highly contagious human pathogen that belongs to the Paramyxoviridae family. ${ }^{1}$ The disease spreads through coughing, sneezing, near contact or direct contact with infected nasal or throat secretions. It has an incubation period located between 9 and 12 days and an infectivity period located between 4 and 9 days. $^{2}$ Deaths due to measles are quite common among malnourished children and people whose immune system has been weakened by diseases that include human immunodeficiency virus/acquired immunodeficiency syndrome (HIV/AIDS). Measles leads to other complications such as blindness, brain swelling (encephalitis), diarrhoea, ear infections and respiratory infection such as pneumonia. High death rates are commonly registered in developing countries with low per capita income and poor health service systems. ${ }^{3}$

Worldwide, measles is ranked among the leading causes of mortality especially among children in developing countries. For instance, in 2013, about 145,700 deaths were recorded. ${ }^{4}$ Until now,

\footnotetext{
* Corresponding author.

E-mail address: dntirampeba@Nust.Na (D. Ntirampeba).
}

there is no antiviral treatment for measles virus. Thus far, measles vaccination and supportive care that includes good nutrition and adequate fluid intake have been used to fight measles. ${ }^{4}$ However, reduction of global funding by the governments and partners has largely affected the immunization campaigns, which hampered efforts for a complete elimination of measles. ${ }^{3}$ Consequently, measles cases are still reported in many countries, with Angola, Ethiopia, Namibia, Bosnia and Herzegovina, Georgia, Sri Lanka and Philippines ranked among the top ten countries with high annualized measles incidence per 100,000 inhabitants in $2014 .^{5}$

In Namibia, as in many countries, diseases surveillance data are often analyzed in the form of aggregated data at health district or regional level because of confidentiality issues. However, health decisions might be needed at lower political boundaries such as constituencies. Nevertheless, direct inference at such lower level made on the basis of regionally aggregated data may lead to spatial misalignment problem. ${ }^{6}$

In brief, spatial misalignment appears through various processes. The first process is when the purpose of the analysis is to make inference about new points based on available information at different points or locations. This is known as point-to-point change of support. The second is when a researcher might be 
interested at predicting values at blocks level using information available at point level. This is called the point-to-block change of support. In the third process, one might seek to make inference from block values to point level, and is referred as the block to point change of support. ${ }^{6}$ In this scenario, it is inappropriate to infer about the relationships between variables at individual level using information observed at area level, as the accuracy at area and point levels is not a one-to-one relationship. This challenge is referred to as ecological fallacy. Fourth, spatial misalignment can arise when the purpose of the spatial analysis is the interpolation at new aggregation level that is different from a level where data were observed. Scholars refer this to as modifiable area unit problem.

Various methods for resolving misalignment have been proposed. $^{7-13}$ For instance, methods have been applied to downscale the distributions of data from coarse to fine grain that include direct method, point sampling method and hierarchical Bayesian method. These methods have been adopted to deal with this scenario of misalignment. ${ }^{8-10}$ Other techniques have been developed that deal with spatial misalignment that arises when the response variable is available at bigger irregular-shaped area units and covariates are available at smaller fine grids, ${ }^{11}$ in which a multi-step approach has been applied. In the case where misalignment occurs with non-nested overlapping grids, hierarchical Bayesian approaches have been employed. ${ }^{6,12}$ Recently, the later has been extensively applied as it permits to derive posterior predictive distributions for both parameters and epidemiological outcome of interest. It is also suitable when dealing with multiple sources of uncertainty and enables to incorporate additional sources of information in the form of prior knowledge. ${ }^{13}$

The aim of this study is two-fold. First, the study aimed to identify constituencies (sub-regions) in Namibia that have elevated risk of measles and also to visualize smoothed patterns of risk of measles. Second, the study aimed to determine factors associated with the risk of measles in Namibia.

\section{Methods}

\subsection{Sources of data}

Measles cases were abstracted from the health management information system (HMIS) database within the Ministry of Health and Social Services (MoHSS) in Namibia. The database included all suspected measles cases from which positive cases were extracted. Any patient consulting a health facility becomes a suspected case if the patient is diagnosed with fever and generalized maculopular rash lasting for three days or longer and cough, coryza or conjunctivitis. Such a case will be investigated and adequate blood specimen is collected and examined at the Namibia Institute of Pathology (NIP). If the blood specimen is found to have serological confirmation of a recent virus infection, the case is classified as laboratory confirmed. However, there are other cases wherein blood specimens are not taken for serological, but are linked to laboratory confirmed cases. Such cases are known as epidemiologically confirmed. A suspected case is discarded if it has been completely investigated or the blood specimen is declared by NIP as not having serological evidence of recent measles virus. This determination of a measles case is based on World Health Organization's (WHO) standard definition, which considers a measles case as either an epidemiologically confirmed case or a laboratory confirmed case. ${ }^{4,5}$ Although the HMIS database has information from 2001 to partly 2015, the 2005-2014 period provided consistent information for the entire country, and hence only data from this period were considered in this study.

Covariates used in this study were obtained from the 2011 Namibia population and housing census (NPHC) and the 2013 Namibia demographic health survey (NDHS). The variables include proxies of social mixing patterns (average household size and proportions of children attending pre-primary and schools), unemployment rates and birth rates. Table 1 provides a list of all variables used in the analysis, as identified through literature. ${ }^{2,14-19}$ Shapefiles that defined the administrative boundary maps were also obtained from the Namibia Statistics Agency (NSA). Although the administrative boundaries have changed over time, in this study we have used the 2011 administrative boundaries that match with variables derived from the 2011 NPHC.

\subsection{Statistical methods}

Each of the 13 regions in Namibia is sub-divided into constituencies, giving a total of 107 sub-regions. The counts of measles cases are available at 13 regions, of which our aim is to estimate the risk at constituency level. This introduces the problem of misalignment in the analysis. To overcome misalignment, two approaches (multi-step and direct methods) are used.

\subsubsection{Multi-step approach}

This method allows the allocation of region/district disease totals to constituency proportional to the constituency area or population. However, the areal area proportional allocation method assumes that population is uniformly distributed throughout the entire area. Namibia is a semi-desert country, and its population is not spread uniformly throughout the territory; rather, people are living in towns or settlements. It has been shown that the measles infection is proportional to the size of the population in each location..$^{2}$ Thus, the population proportional allocation was applied. The steps of multi-step method are as follows:

(i) Overlay constituencies on regions. This enables to determine exactly what proportion of a given constituency is susceptible or infected by measles.

(ii) Find all total values of measles cases for all constituencies. The computation of these values is based on population proportional allocation concept, as it has been shown to be more appropriate relative to areal proportional allocation for infectious diseases. ${ }^{2}$ This is formulated as follows: $y_{i k}=$ $\frac{P_{i k}}{P_{k}} Y_{k}$, where $y_{i k}$ is the number of measles cases in the $i$ th

Table 1

Description of variables considered for the analysis.

\begin{tabular}{|c|c|}
\hline Variable & Variable name \\
\hline 1 & Standardized average household size \\
\hline 2 & Counts of measles for previous year (2004) \\
\hline 3 & Unemployment rates \\
\hline 4 & Standardized birth rates \\
\hline 5 & Proportion of children attending schools \\
\hline 6 & Proportion of vaccinated children against measles by age 12 months \\
\hline 7 & Proportion of children attending pre-primary \\
\hline
\end{tabular}


constituency of region $k ; Y_{k}$ is the number of measles cases in the $k$ th region that contains the constituency $i ; P_{i k}$ is the total population of constituency $i$ included in the region $k$; and $P_{k}$ is the total population of the region $k$.

(iii) Apply spatial smoothing techniques to the computed measles cases. To this end, we explored the Poisson and negative binomial hierarchical regression modelling approach. These models are ideal for count data, and by applying hierarchical models, we incorporate covariate information and any other sources of uncertainty in parameter estimation process.

The model formulation given below follows the formulation of nested block-level modelling developed by Banerjee. ${ }^{6}$ In brief, let $I$ be the number of constituencies overlaid in a given region and $K$ be the number regions in Namibia; $y_{i k}$ is the total measles count in the constituency $i$ of region $k$ such that $i=1,2, \ldots, I, n_{i k}$ and $k=1,2$, $\ldots, K ; n_{i k}$, is the population count in constituency and $\lambda$ is the probability of contracting the disease. In this study, $\lambda$ is assumed to be the 2014 Namibia annualized measles incidence per 100,000 inhabitants such that $E_{i k}=\lambda n_{i k}$ is the corresponding expected disease count for constituency $i, m_{i k}$ is the relative risk for contracting the disease in the constituency $i$ and $x_{i k}$ are covariates present in constituency $i$.

The first-stage model for disease counts is given by a Poisson such that

$y_{i k} \mid m_{i k} \sim \operatorname{Poisson}\left(E_{i k}, m_{i k}\right)$

$\log \left(m_{i k}\right)=\log E_{i k}+X_{i k}^{T} \beta+\phi_{i k}+w_{i k}$,

where in Eq. (2) we specify the distribution of $m_{i k}$ as a function of the covariates $\left(X_{i k}^{T}\right)$ in the constituency $i$ for some fixed effects, $\beta$, and spatial random effects, $\phi_{i k}$ and $w_{i k}$.

Alternatively, a negative binomial (NB) can be specified as

$y_{i k} \sim N B(n, p)$

$p=\frac{\exp \left(X_{i k}^{T} \beta+\phi_{i k}+w_{i k}\right)}{1+\exp \left(X_{i k}^{T} \beta+\phi_{i k}+w_{i k}\right)}$

for $y_{i k}=0,1,2, \ldots$ and $n_{i k}$ is the number of successive trials with a probability of success $p$. Similarly, in Eq. (4), $p$ is modelled as a function of covariates and some spatial random effects in similar way as in Eq. (2).

Estimation of the models (2) and (4) follows a Bayesian inference approach. As such, prior assumptions need to be specified. For fixed effect parameters $\beta$, weak informative Gaussian priors $\beta \sim N\left(0, \tau^{-1} I\right)$ with small precision $\tau$ on identity matrix will be assumed. The term $\phi_{i k}$ assumes a prior normal $N\left(0,1 / \tau_{h}\right)$ and it controls the global extra-variability in the relative risks or captures the constituency-wide heterogeneity. This prior is also referred to as the independent and identically distributed (IID)prior, such that the effect $\phi_{i k}$ for each constituency is independent of all other constituencies.

On the other hand, the component $w_{i k}$ captures the extraPoisson dispersion in relative risks that vary locally (spatial clustering) and $w_{i k}$ are assumed to be distributed according to intrinsic conditional autoregressive (ICAR) model. We specify the ICAR model as follows:

$w_{i k} \mid w_{i k \neq j} \sim N\left(\frac{1}{n_{i}} \sum_{i k} w_{i k}, 1 / \tau_{n_{i}}\right)$,

where $\tau$ and $n_{i}$ are the precision parameter and the number of neighbours of constituency $j .{ }^{20}$ Under this prior, the effect of $w_{i k}$ for each constituency $i$ is normally distributed with mean effect equalling the average effect of effects of neighbours of constituency $i$ with $1 / \tau_{n_{i}}$ precision. The neighbours are defined in terms of constituencies sharing at least one point (queen adjacency).

\subsubsection{Direct approach}

This is one of the models that is commonly used in ecology to downscale the distribution of species from coarse scale to fine scale. ${ }^{8-10}$ The conventionally used direct approach ${ }^{8,10}$ assumes that the cases' distribution at fine scale (constituency level) is driven by the same processes as at coarse scale (regional/district level). Thus, the method fits a hierarchical spatial regression model at coarse scale and then the estimated parameters are used in the spatial regression at fine scale.

Specifically, in this study, we fit the following model:

$y_{i} \mid m_{i} \sim \operatorname{Poisson}\left(E_{i} m_{i}\right)$

$\log \left(m_{i}\right)=X_{i}^{T} \beta+\emptyset_{i}+w_{i}$

where in model (7) the distribution of the relative risk $m_{i}$ was specified as a function of the covariates $X_{i}^{T}$ (mean values of covariates at constituency level) in the region $i$ for some fixed effects, $\beta$, and some spatial random effects, $\emptyset_{i}$, and $w_{i}$. The prior distributions were specified in the same way as in the aforementioned multi-step approach.

The fixed effects $\beta$ are then used directly in Eq. (8) in order to predict the specific constituency relative risk $m_{i k}$ through the following equation:

$\log \left(m_{i k}\right)=X_{i k}^{T} \beta$.

To predict the number of cases in a given constituency (finescale level), values obtained from model (8) will be fed in the following model:

$y_{i k} \mid m_{i k} \sim \operatorname{Poisson}\left(E_{i k} m_{i k}\right)$

\subsection{Analysis of measles data}

Before fitting the models to the data, we explored the issue of multicollinearity that may arise in the data. Multicollinearity is a condition that happens when independent variables are highly correlated. This condition affects the estimated regression coefficients of independent variables, as their sampling errors tend to be large. Many scholars still insist that there is no clear critical value of correlation among independent variables to signal multicollinearity. ${ }^{21}$ A common way to measure multicollinearity is to use the variance inflation factor. Generally, the multicollinearity between independent variables is regarded to be severe if the largest variance inflation factor is greater than 10 .

To avoid multicollinearity, we ran a correlation analysis for the variables (Table 1) and computed the variance inflation factor. It was found that the variables standardized average household size and proportions of children attending schools $(\rho=0.652)$, proportions of children attending schools and proportions of children attending pre-primary $(\rho=0.6452)$, and standardized average household size and proportions of children attending pre-primary $(\rho=0.552)$ were highly correlated. The variance inflation factor was 11.5 . Consequently, only the variable standardized average household size as a proxy of social mixing was used.

We then fitted 12 models, which are summarized in Table 2 . The first three models are for both Poisson and negative binomial distributions that assume the variability in measles incidences is solely due to spatial random effects. 
Table 2

Summary of models fitted to measles data for Namibia and their corresponding DICs.

\begin{tabular}{clllll}
\hline \multicolumn{2}{c}{ Spatial component } & Fixeed & $\overline{D(\mu)}$ & $p_{D}$ & DIC \\
\hline Poisson model & & & & \\
1 & IID & & 657.58 & 99.43 & 856.44 \\
2 & ICAR(BESAG) & & 661.07 & 94.44 & 849.95 \\
3 & CAR(BYM) & All covariates & 658.15 & 97.93 & 854.01 \\
4 & IID & All covariates & 662.3 & 93.27 & 848.84 \\
5 & ICAR(BESAG) & All covariates & 662.4 & 93.29 & 848.94 \\
6 & CAR(BYM) & & & & \\
Negative Binomial model & & 1081.72 & 1.96 & 1085.64 \\
1 & IID & & 939.14 & 43.15 & 1025.44 \\
2 & ICAR(BESAG) & & 938.78 & 43.42 & 1025.62 \\
3 & CAR(BYM) & All covariates & 1060.39 & 6.93 & 1074.25 \\
4 & IID & All covariates & 939.47 & 42.06 & 1023.59 \\
5 & ICAR(BESAG) & All covariates & 941.41 & 41.16 & 1023.73 \\
6 & CAR(BYM) & A & &
\end{tabular}

DIC: deviance information criteria; all covariates, covariates are described in Table 1.

Model 1 explores only the IID random effects. Model 2 assumes for each region a spatially structured random effect that is distributed, through ICAR, and called the Besag model. The third model (Model 3) is convolution model that assumes for each region two components of random effect, namely, unstructured IID and ICAR; in other words, this is a Besag-York-Mollie (BYM) model. The other three models for both distributions were obtained by adding covariates to models 1-3 in order to assess effects of covariates on the risk of measles. The best model was identified using the deviance information criterion (DIC). The DIC is given by $=D+2 p$, where $\mathrm{D}$ the deviance is evaluated at the posterior mean and $\mathrm{p}$ is effective number of parameters. By the rule of thumb, the best model is one with the smallest DIC.

The significance of parameters was assessed using credible intervals. Generally, if a credible interval for $\theta$ does not contain zero, then the parameter is statistically significant. In Bayesian setting, a $100(1-\alpha) \%$ credible interval for $\theta$ is an interval $(a, b)$ such that $\left(a \leq \theta \leq b \mid o_{1}, \ldots, o_{n}\right)>(1-\alpha) 100 \%$, where $\alpha$ is a small value between 0 and 1 , and $o_{1}, \ldots, o_{n}$ are observed sample values. It is the analogue of confidence interval in classical approach. When data have been observed, the credible interval is fixed while $\theta$ is random. This is in dissimilarity to the classical confidence interval where the interval is random while $\theta$ is a fixed parameter. The interpretation of a credible interval is different from the one of classical interval. In the Bayesian paradigm, the credible interval is interpreted as "the probability is at least $(1-\alpha) 100 \%$ that $\theta$ lies within the interval $(a, b)$ ". In classical approach, the confidence interval is interpreted as " $(1-\alpha) 100 \%$ of all such intervals $a, b$ will contain the true parameter $\theta$."

To estimate Bayesian posterior marginal distributions and any other posterior inferences for all the 12 models, the integrated nested Laplace approximation (INLA) approach was used. Model fitting was carried out in R statistical software.

\section{Results}

\subsection{Model selection}

Table 2 shows that Models 2 and 3 (Poisson models), which took into account the random spatial variation, are the best competing models (DICs: 849.95 and 850.07, respectively) among those that did not include covariates. This reveals the presence of spatial clustering or correlation in measles risk. After adding covariates in models, it was noted that Models 5 and 6 were equally good fitting for this data. Model 5 was used to generate the relative risks (RR) for contracting measles virus and probabilities to assess constituencies with elevated relative risks.

\subsection{Fixed effects}

Table 3 presents a summary of fixed effects for all variables included in the model. In summary, from $95 \%$ credible intervals, we observed that the standardized birth rates, counts of measles for previous year (2004) and unemployment rates had significant positive effects on measles incidence, whereas the proportion of vaccinated children against measles by age 12 months had significant negative association with risk of measles. However, the standardized average household size did not show any significant association, although this was positively related to the risk of measles.

\subsection{Spatial distribution of measles relative risks}

In disease mapping, the most important aspect is to determine the areas with excess risks. Maps (a) and (b) in Fig. 1 show the distributions of constituency specific relative risks obtained from the multi-step approach and direct approach, respectively. Map (a) indicated that the Kunene region has constituencies with high residual relative risks, whereas map (b) showed that Epupa (Kunene), Mungu and Mukwe (Kavango), Guinas and Tsumeb (Oshikoto) and Omatako and Okahandja (Otjzondjupa) constituencies had high relatives.

\subsection{Modelling approach comparison}

While the multi-step modelling approach provides a DIC statistic, the direct method does not provide such statistic. Thus, the comparison of the models resulting from these modelling approaches cannot be achieved through a DIC statistic. The analysis of residuals has been used as an exploratory tool to assess which model performs better. Fig. 2 gives a boxplot of standardized residuals obtained from the direct and multi-step approach models, respectively. This figure indicates that the multi-step approach model provides lower median standardized residuals. In addition, the models were compared using the mean root square error (MSRE) statistic. It was found the MSRE associated with the multi-step approach model was small relative to one of the direct models (i.e. 6.40 versus 6.60 ), thus confirming the boxplot results

Table 3

Summary statistics: fixed effects (posterior mean), posterior standard deviation and posterior 95\% credible interval for Model 5 .

\begin{tabular}{|c|c|c|c|}
\hline Fixed effects & Posterior mean & Standard deviation & $95 \%$ credible interval \\
\hline Standardized average household size & 0.0523 & 0.063 & $(-0.072,0.176)$ \\
\hline Counts of measles for previous year (2004) & 0.017 & 0.006 & $(0.0043,0.0294)$ \\
\hline Unemployment rates & 0.007 & 0.003 & $(0.0011,0.0129)$ \\
\hline Standardized birth rates & 0.143 & 0.048 & $(0.0494,0.2364)$ \\
\hline Proportion of vaccinated children against measles by age 12 months & -0.0050 & 0.003 & $(-0.0099,-0.0001)$ \\
\hline
\end{tabular}


(a)

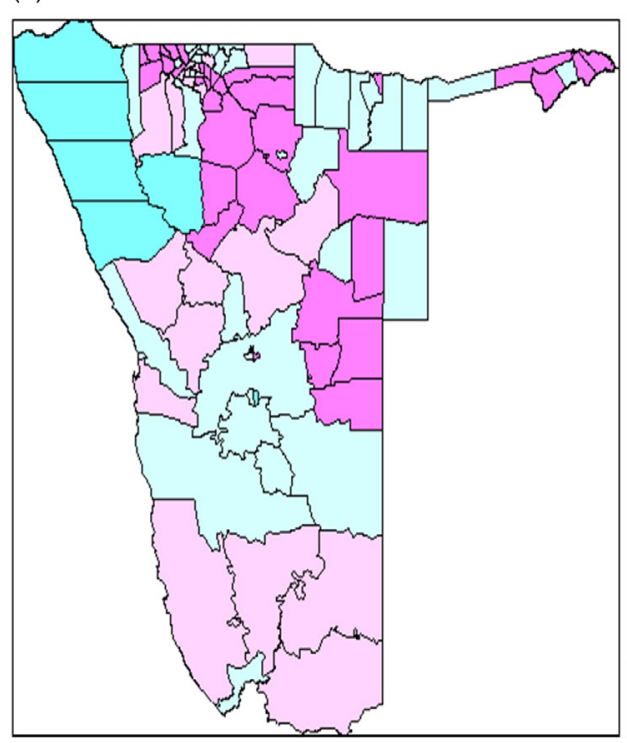

(b)

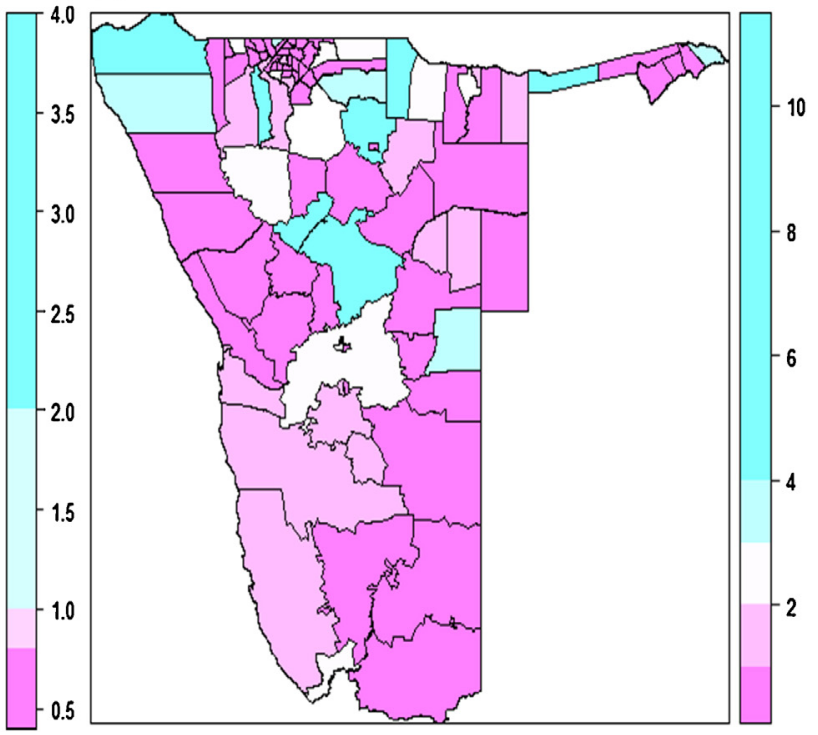

Fig. 1. Distribution of constituency specific relative risks (SRR) of measles (multi-step approach) (a); specific relative risks (SRR) of measles (direct approach) (b).

that the multi-step approach model provided a relatively better model.

\section{Discussion}

The main aim of this study was to use aggregated data obtained at regional level to estimate and map the risk of measles at a lower level (constituency level). To achieve this, we corrected for spatial misalignment using both direct and multi-step approach methods. Subsequently, a spatial Poisson regression model was applied to explain the variation of measles risk in Namibia. The model thus developed included socio-economic covariates that explained the risks of measles in Namibia.

Findings show that the measles risk varied remarkably (Fig. 1). Using either the direct or multi-step approaches, constituencies of high risk were observed along the borders with Angola, notably in Kunene region (i.e. Opuwo, Sesfontein, Khorixas, Kamanjab and Outjo constituencies) and Kavango region (i.e. Epupa, Mukwe and Mpungu constituencies). This could be due to free movement to and from Angola, whereby visits to Angola may expose the nonimmunized to the disease. ${ }^{14,15}$ In addition, using the multi-step approach, high-risk areas can be identified in Hardap and Ohangwena regions. Regular surveillance of population movement may assist in controlling the risk of the disease, particularly regular

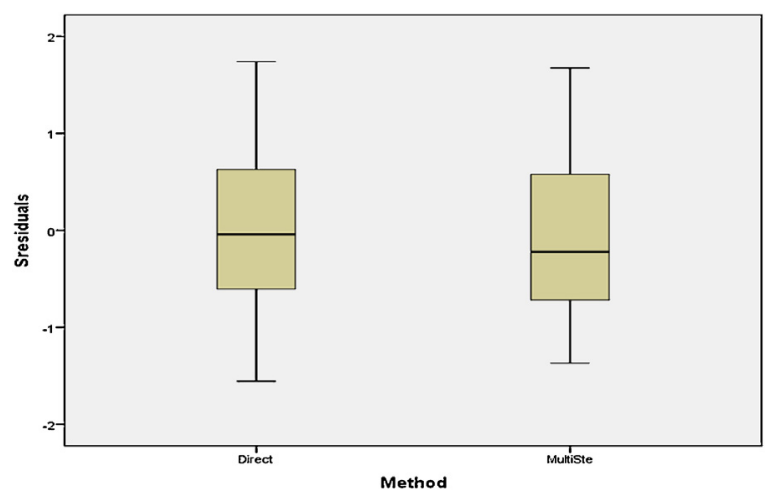

Fig. 2. Boxplot of standardized residuals obtained from the two modelling approaches (at regional level). border checks and targeted vaccination of children in the areas identified as high risk or along all border areas.

Furthermore, results show that covariates like the standardized average household size, previous counts of measles, standardized birth rates and unemployment rates were associated with increased measles risk. Such findings are similar to what was obtained elsewhere, and is typical of contagious diseases like measles, 2,10,14,17,18,24 and confirm that contact is critical at sustaining transmission - and that large households are at increased risk. ${ }^{10,24}$ Existing reservoirs of the disease are a major source for maintaining the transmission to the subsequent year. Any surveillance programme should try to eliminate as much as possible any putative source of transmission. Further, there is clear evidence that poor households and neighbourhoods, as measured by unemployment rate, are the most vulnerable. Pathways of transmission are not quite clear within poor households and neighbourhoods, but may reflect heightened contacts of the infected and the susceptible, thus fuelling multiple infections.

We also found that vaccination reduced the risk of the disease. In fact, vaccination coverage is reported to be $90 \%$ among children aged $12-23$ months countrywide. ${ }^{25}$ It is therefore imperative that existing policies such as supplementary vaccination campaign every three years or in case of measles outbreaks in Namibia should be maintained. Namibia may need to improve the delivery of measles vaccine by for example borrowing and improving the standard protocol of systematic reminder/recall interventions by telephone or post that has been proven to be an effective strategy in increasing measles vaccine coverage. ${ }^{26}$ Otherwise, improper vaccination procedures and any other vaccine-related factors may cause the resurgence of measles. ${ }^{17}$ The failure to vaccine all susceptible persons remains an obstacle to measles elimination, as studies have shown that measles virus can still travel along the chains of transmission among vaccinated persons and infect unvaccinated people or people who have not acquired immunity by recovering from the disease. ${ }^{24}$

Studies have further shown that care-seeking patterns are among the reasons of missing measles vaccination and underreporting of measles cases. ${ }^{17,26}$ Although the existence of factors that may affect care-seeking patterns is acknowledged within the MoHSS, in Namibia, there are no data documenting factors affecting care seeking. Thus, there is a need of a study on care- 
seeking patterns for measles, which can inform better strategies of measles control.

A number of significant weaknesses of this study are acknowledged. Firstly, the use of aggregated data over the period of 20052014 would not allow the observation of any possible seasonality effects, which are quite common in infectious and contagious diseases like measles. Thus, the temporal effects were implicitly masked. Secondly, the accuracy of health information data constitutes a major concern to some extent. Currently, the HMIS database in the Ministry of Health and Social Services does not integrate data from all the MoHSS programmes and it does not routinely capture some critical child programme data. ${ }^{23}$ In addition, the accuracy of information may depend on the level of utilization of health facilities, which in turn is influenced by the accessibility, perceived health service quality and health careseeking behaviour among many other factors. ${ }^{16,22,23}$ As a result, many cases of measles are never reported to the health management system. Such under-reporting may somehow distort the geographical pattern of disease risk. ${ }^{26}$ Nevertheless, spatial smoothing approach used in this study may have attenuated an aberrant. $^{2,11}$ Thirdly, this study has assumed that the covariates did not change in the period of 10 years. Thus, the interpretation of the study findings should take into account this limitation.

In conclusion, the epidemiological implication of this study is that regional aggregated data may present a useful data for policy and decision making at lower level, provided appropriate statistical models are developed and applied. This presents an important tool for the health sector to plan, evaluate and redesign prevention and control strategies, and make important policy decisions particularly for geographically targeted intervention in resource pair settings. With regard to the statistical models presented here, particularly for the multi-step approach, many extensions to the fitted model are possible and include those that can account for the temporal effects and measurement errors.

\section{Authors' contributions}

DN analyzed the data and drafted the manuscript. LNK and IN led and coordinated the study and critically reviewed the manuscript to insure intellectual content. All authors contributed to the conception of the methodological aspect to be followed in the analysis of the data. In addition, all authors read and approved the final manuscript.

\section{Ethics approval}

The Ministry of Health and Social Services has granted us the permission to use the measles dataset.

\section{Availability of data and materials}

The datasets analyzed during in this study are not publicly available due to confidentiality reason but are available from the Ministry of Health and Social Services on reasonable request.

\section{Conflicts of interest}

The authors have none to declare.

\section{Acknowledgements}

The authors thank the Ministry of Health and Social Services for providing us with the measles dataset used in this study.

\section{References}

1. Bhella D, Bourhis JM, Combe CR, et al. Measles Virus Nucleoprotein. New York: Nova Science; 2007.

2. Doungmo Goufo EF, Oukouomi Noutchie SC, Mugisha S. A fractional SEIR epidemic model for spatial and temporal spread of measles in metapopulations. Abstract and Applied Analysis (Vol. 2014). Hindawi Publishing Corporation; 2014, June 22.

3. WHO. WHO Warns That Progress Towards Eliminating Measles has Stalled. Available from: http://www.who.int/mediacentere/news/release/2014/ eliminating-measles/en/ [cited 23.01.17].

4. WHO. Measles. Available from: http://www.who.int/mediacentre/factsheets/ fs28/en [cited 23.01.17].

5. WHO. Reported Measles Cases and Incidence Rates by WHO Member States 2013, 2014 as of 11 February 2015. Available from: http://www.who.int/ immunization/.../measlesreportedcasesbycountry.pdf [cited 23.01.17].

6. Banerjee S, Carlin BP, Gelfand AE. Hierarchical Modeling and Analysis of Spatial Data. New York: Chapman \& Hall; 2004.

7. Goovaerts P. Kriging and semivariogram deconvolution in the presence of irregular geographical units. Math Geosci. 2008;40(January (1)):101-128.

8. Keil P, Belmaker J, Wilson AM, Unitt P, Jetz W. Downscaling of species distribution models: a hierarchical approach. Methods Ecol Evol. 2013;4 (January (1)):82-94.

9. Sturrock HJ, Cohen JM, Keil P, et al. Fine-scale malaria risk mapping from routine aggregated case data. Malar J. 2014;13(November (1)):1.

10. Araújo MB, Thuiller W, Williams PH, Reginster I. Downscaling European species atlas distributions to a finer resolution: implications for conservation planning. Global Ecol Biogeogr. 2005;14(January (1)):17-30.

11. Lee D, Sarran C. Controlling for unmeasured confounding and spatial misalignment in long-term air pollution and health studies. Environmetrics. 2015;26(November (7)):477-487.

12. Finley AO, Banerjee S, Cook BD. Bayesian hierarchical models for spatially misaligned data in R. Methods Ecol Evol. 2014;5(June (6)):514-523.

13. Illian JB, Møller J, Waagepetersen RP. Hierarchical spatial point process analysis for a plant community with high biodiversity. Environ Ecol Stat. 2009;16(September (3)):389-405.

14. Zagheni E, Billari FC, Manfredi P, Melegaro A, Mossong J, Edmunds WJ. Using time-use data to parameterize models for the spread of close-contact infectious diseases. Am J Epidemiol. 2008;168(November (9)):1082-1090.

15. Held L, Höhle M, Hofmann M. A statistical framework for the analysis of multivariate infectious disease surveillance counts. Stat Model. 2005;5 (October (3)):187-199.

16. Adika OV, Baralate S, Agada JJ, Nneoma N. Mothers perceived cause and health seeking behaviour of childhood measles in Bayelsa, Nigeria. J Res Nurs Midwifery. 2013;2:6-12.

17. Jasem J, Marof K, Nawar A, Islam KM. Epidemiological analysis of measles and evaluation of measles surveillance system performance in Iraq, 2005-2010. Int J Infect Dis. 2012;16(March (3)):e166-e171.

18. Mayet A, Genicon C, Duron S, et al. The measles outbreak in the French military forces - 2010-2011: results of epidemiological surveillance. J Infect. 2013;66 (March (3)):271-277.

19. Beyene BB, Tegegne AA, Wayessa DJ, Enqueselassie F. National measles surveillance data analysis, 2005 to 2009, Ethiopia. J Public Health Epidemiol. 2016;8(March (3)):27-37.

20. Besag J, York J, Mollié A. Bayesian image restoration, with two applications in spatial statistics. Ann Inst Stat Math. 1991;43(March (1)):1-20.

21. Keller G. Managerial Statistics. Mason, OH: South-western; 2012.

22. Ministry of Health and Social Services (MOHSS). Namibia Child Survival Strategy 2014-2018.

23. De Savigny D, Mayombana C, Mwageni E, et al. Care-seeking patterns for fatal malaria in Tanzania. Malar J. 2004;3(July (1)):1.

24. Ma C, Hao L, Zhang Y, et al. Monitoring progress towards the elimination of measles in China: an analysis of measles surveillance data. Bull World Health Organ. 2014;92(May (5)):340-347.

25. Ministry of Health and Social Services (MOHSS). Namibia Demographic Health Survey 2013

26. Filia A, Bella A, Rota MC, et al. Analysis of national measles surveillance data in Italy from October 2010 to December 2011 and priorities for reaching the 2015 measles elimination goal. Korea (in 2006). 2013;1(May):4-5. 